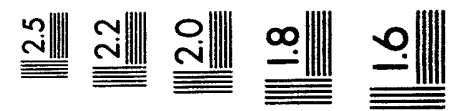

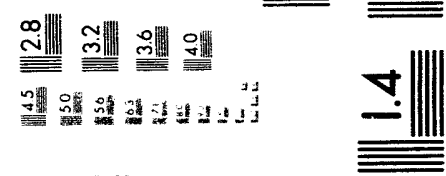

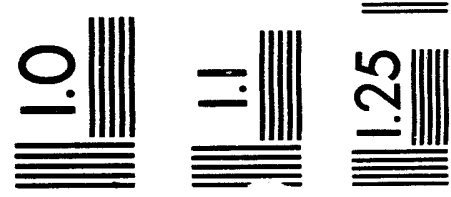



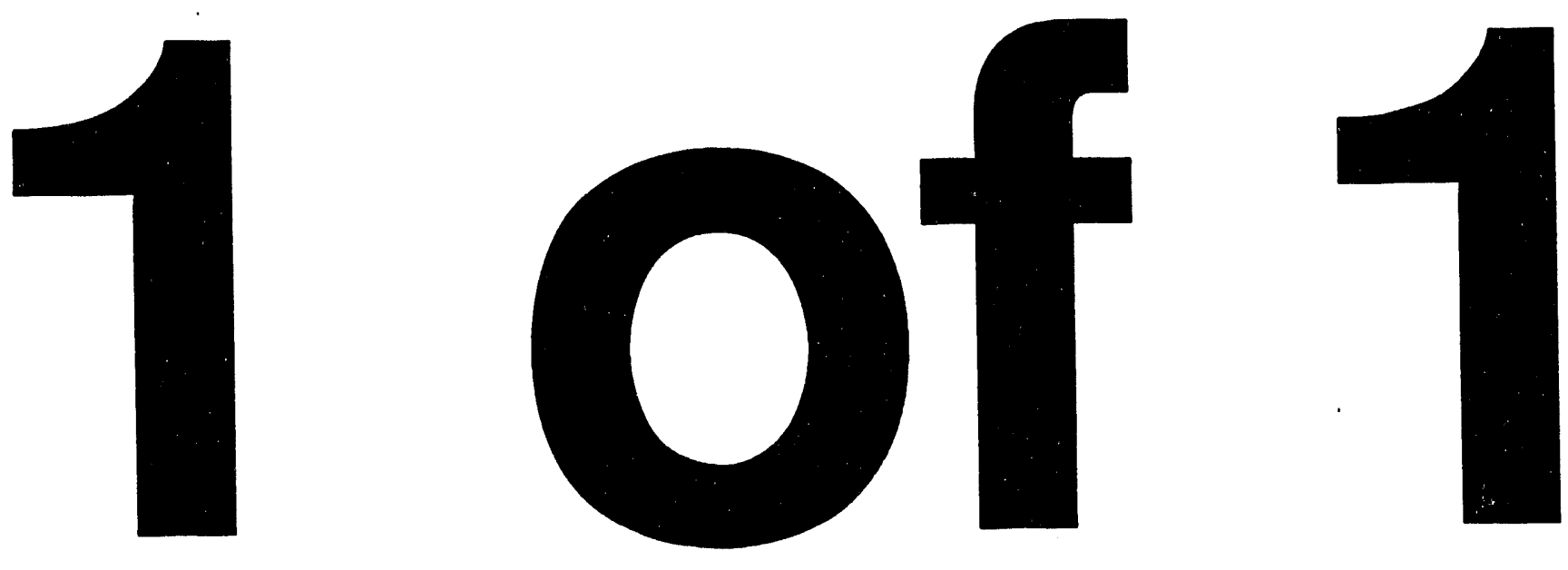


\title{
Survey of Hardware Supported by the Control System at the Advanced Photon Source*
}

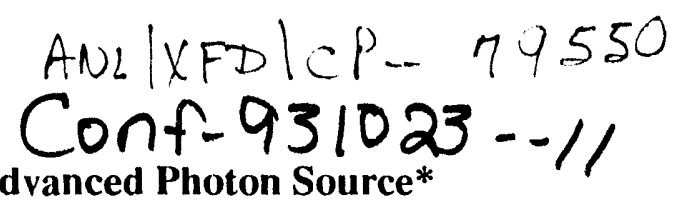

\author{
K.J. Coulter, G.J. Nawrocki \\ Argonne National Laboratory \\ Advanced Photon Source \\ 9700 South Cass Avenue \\ Argonne, Illinois 60439
}

\section{Abstract}

The Experimental Physics and Industrial Control System (EPICS) has been under development at Los Alamos and Argonne National Laboratories for over six years. A wide variety of instrumentation is now supported. This presentation will give an overview of the types of hardware and subsystems which are currently supported and will discuss future plans for addressing additional hardware requirements at the APS. Supported systems to be discussed include: motion control, vacuum pump control and system monitoring, standard laboratory instrumentation (ADCs, DVMs, pulse generators, etc.), image processing, discrete binary and analog $\mathrm{I} O \mathrm{O}$, and standard temperature, pressure and flow monitoring.

\section{INTRODUCTION}

The Experimental Physics and Industrial Control System (EPICS) is currently being implemented to control all accelerator subsystems at the Advanced Photon Source (APS) at Argonne National Laboratory. In order to address the different needs of all of these subsystems, EPICS must support a wide variety of instrumentation on several different hardware buses. This paper briefly summarizes the major hardware systems already in use at the APS; it does not attempt to cover all instrumentation interfaced toEPICS. The instrumentation described in this paper covers motion control systems, vacuum pump controllers and system monitoring, standard laboratory instruments, image processing systems, discrete binary and analog $1 / O$ modules, and standard temperature, pressure, and flow monitoring devices.

\section{CONTROL SYSTEM ARCHITECTURE AND SUBNETS}

The APS control system provides for VME-based Input/ Output Controllers (IOCs) to be distributed throughout the facility and interconnected via Ethernet to one another and also to UNIX-based Operator Interface (OPI) consoles. Instruments with the VME-bus interface reside directly in the IOCs. VXIbus instruments are supported through VME-VXI extenders from the IOCs. The IOCs also support VME interfaces to various types of message based I/O such as GPIB. Although this distributed architecture allows for intelligent processors to be near the major subsystems, cost and environmental factors often impact the ability to place IOCs near the controlled hardware.

\footnotetext{
*Work supported by U.S. Department of Energy, Office of Basic

Energy Sciences under Contract No. W-31-109-ENG- 38.
}

Therefore, $\mathrm{I} / \mathrm{O}$ subnets are frequently required to interface directly to the equipment and communicate $\mathrm{I} / \mathrm{O}$ information to the nearest IOC. [1]

One such subnet utilizes the Allen-Bradley 1771 series $1 / O$ modules to provide remote interfacing for discrete binary and analog types of signals. This subnet is implemented in the APS control system via either "twisted pair" or optical fibers. Although effective, it is best used for a multitude of signals as its use becomes cost prohibitive when used with just a few raw binary or analog signal points. An additional complication with the Allen-Bradley solution is that the network and I/O chassis are proprietary designs of Allen-Bradley. Modules cannot be customized to meet additional subnet requirements such as an interface for GPIB and RS232 signals. [2]

To provide an additional subnet interface for the APS control system a "gateway" for GPIB, RS232, and raw binary and analog I/O signals was implemented using the BITBUS † protocol. The BITBUS subnet is based upon a non-proprietary commonly accepted network which provides the ability to communicate control signals to multiple nodes, over distances up to several hundred meters, and in electrically harsh environments. To implement the BITBUS subnet in the APS control system, a BITBUS slave node was developed, this instrument has taken the form of the BITBUS Universal Gateway, or BUG. The BITBUS subnet interface to the APS control system is accomplished through the BITBUS master. The master is a modified BITBUS node with an interface to the VME host computer bus. Currently the BITBUS subnet master in the APS control system is either the Xycom/IST XVME-402 VME module, or the PEP Modular VMOD-2 with up to two BITBUS master daughter cards. Both of the cards are modified for use with the APS BITBUS optical fiber subnet. [2]

\section{MOTION CONTROL}

EPICS currently has capabilities for both open-loop and closed-loop motion control systems using the Oregon Micro Systems (OMS) VMEbus family of stepper motor controllers. Four-, six-, and eight-axis controllers are all supported; the four-axis controller will also accept up to 4 axes of incremental encoder feedback (using TTL quadrature output signals). Absolute encoders are supported via the VAROC VME Absolute Encoder Interface board from the European Synchrotron Radiation Facility. This board interfaces up to 16 absolute encoders using the synchronous serial interface. EPICS support for motion control allows the feedback loop to be closed with any EPICS data value; feedback is not limited to encoders, but can come from any instrument or combination of instruments under EPICS control. 
The loop is closed through EPICS software links, which have more than sufficient response times for motion control applications. The software support for motion control allows for user specification of coordinate transformations, PID loops, deadband definitions, positioning retry limits, and in the future will have backlash correction. The EPICS motion control support is described more fully in reference [3].

\section{VACUUM PUMP CONTROL AND SYSTEM MONITORING}

The majority of the vacuum system control and monitoring devices are RS232 or GPIB controlled instruments. This Includes the Digitel 500 Ion Pump Coniroller (RS232 supported only) and the Granville Phillips GP307 Vacuum Gauge Controller (RS232 and GPIB supported). The Granville Phillips GP307 Vacuum Gauge Controller can also be equipped with a custom made fiber optic BITBUS interface. This interface was developed to provide greater functionality than included with the standard RS232 or GPIB interfaces, and connects to the existing BITBUS subnet. The Vacuum Valve Controller is also custom built for the unique requirements of the APS. This unit is normally equipped with a custom fiber optic BITBUS interface, which of course attaches to the BITBUS subnet. It can, however, be fit with a different control module for use with industry standard 24V DC signals for basic binary control and monitoring. Currently residual gas analyzer (RGA) device support is under development. It is intended to support the Spectra Multi-Quad family of RGA devices.

\section{STANDARD LABORATORY INSTRUMENTATION}

Currently the majority of standard laboratory instrumentation used at the APS (oscilloscopes, pulse generators, power supplies, etc...) are interfaced to the control system with GPIB (IEEE-488) or RS232 interfaces. If a GPIB instrument is located close to a control system IOC, or requires frequent large data transactions with the control system, it is often interfaced via a National Instruments GPIB 1014 VME card. This card provides a single GPIB port interface to the IOC for control and monitoring of up to 15 GPIB instruments. More often, however, GPIB instruments are placed too far away from an IOC for the GPIB electrical specification. Although both fiber optic and twisted pair extenders are available for GPIB they are cost inefficient. In this case GPIB instruments ace interfaced to the control system via BUGs on the BITBUS subnet. [2] RS232 instruments are interfaced to the APS control system exclusively via BUGs. The obvious reasons for this are that RS232 offers no ground isolation, severe distance limitations, and exists with a single node master/slave topology. Again, extenders and multidrop RS232 network solutions are available, however, their repeated use can be expensive. Another reason is that RS232 instruments are often very different in both their command sets and how they respond to commands. For instance, some instruments may or may not echo each received byte back to the control computer. Some units respond to commands after a carriage return, others need the command placed in parentheses. A variety of instruments return data with a carriage return and/or line feed, others use odd termination or data return framing characters. For these specialized cases, unique software for each type of controlled instrument may be created for the BUG. Despite this additional software design work, there are distinct advantages to allowing a universal version of RS232 software to reside at the point of the control system IOC. The BUG can also be given the ability to remove worthless data bytes or interpret data sent by an RS232 instrument into a more concise package to be sent to the control system. For these reasons, the RS232 BUG is currently the only recommended method for RS232 instrument interfacing to the control system. [2] Future plans for message based $1 / 0$ include the use of the Motorola MVME 162 Intelligent IndustryPack carrier module. This is a VME module with a 68040 micro processor, and IndustryPack expansion interfaces. These expansion interfaces are capable of holding any combination of four $\mathrm{I} / \mathrm{O}$ modules. The current plan is to use the 68040 on the MVME 162 as an auxiliary processor to handle the massive processing overhead common with such message based transactions. The four IndustryPack expansion slots could be configured to provide any combination of GPIB port, dual port RS232 interface, and BITBUS master.

There are a multitude of device support modules written for various GPIB instruments, and a few for RS232 instruments via the BUG. Device support libraries and example modules are set up in such a way that it is not difficult for an application developer to create additional device support modules if support is required for additional instruments.

\section{IMAGE PROCESSING}

Development is currently underway for providing image processing capabilities in EPICS using a Datacube Inc. Max Video 20 VME bus card. The Max Video 20 is a 2-slot VME card with $6 \mathrm{MB}$ memory and 8-bit digitizing for up to $1024 \times 1024$ pixels operating at a maximum rate of $26 \mathrm{MHz}$. The Max Video 20 is fully configurable to match any standard $30 \mathrm{~Hz}$ camera. The video data, an $x$ or $y$ profile, and text overlays are displayed realtime on a dedicated display screen. The Max Video 20 offers a user-definable region of interest, real-time background subtraction, edge detection, and feature listings (the $\mathrm{x}$ and $\mathrm{y}$ coordinates of pixels greater than some user-defined value). The data resides in dual-ported memory, so that it is available to both the display, and to the VMEbus memory for manipulation by other resources. Currently, centroid and RMS calculations are carried out in software and sent back to the Max Video to be overlaid on the display.

Additional EPICS functionally that will be added for image processing include adding pseudo-color to the displays, the ability to save and restore specific images, supporting calibration marks and values, and simplifying the user input for defining the Max Video 20 setup. The Max Video 200 will soon be available. It will have $24 \mathrm{MB}$ memory, allow real-time display of both $x$ and $y$ profiles, sum of all pixels, sum squared and other statistics.

\section{DISCRETE BINARY AND ANALOG L/O}

Among the most common types of signals in an accelerator control system are the discrete binary and analog $\mathrm{I} / \mathrm{O}$ signals. If 
these signals are located within close proximity to an IOC, they may be interfaced directly to a selection of supported VME cards. For binary signals, supported are the Xycom XVME-210 and 220 . These modules provide 32 channels of optically isolated binary inputs and binary outputs respectively. A new addition is the Acromag 9440 series modules which provide for 16 optically isolated binary inputs and 16 optically isolated binary outputs on a single module. This module is also convenient because it offers front panel LEDs which indicate the status of each channel.

Analog signals are supported by the Xycom XVME-566 analog input module, the Analogic DVX 2502 High Speed Analog Input module, and the VMIC VMIVME-4100 Analog output module. The Xycom board accepts 32 single ended or 16 differentially wired analog inputs. It has a 12 bit accuracy and is able to sample at a rate of $100 \mathrm{kHz}$. The VMIC module provides 16 single ended analog outputs and has a 12 bit accuracy, with a maximum settling time of $8 \mu$ s. Faster analog signals are interfaced via the Analogic module. This module accepts up to 8 shielded, differentially wired inputs. It has a 16 bit resolution and may be sampled at a rate of $200 \mathrm{kHz}$.

Subnet interfacing of remote binary and analog signals may be provided by the BUG equipped with a custom designed iSBX $\dagger$ (single board extension) bus $\mathrm{I} / \mathrm{O}$ board, or the AllenBradley 1771 series of modules. The BUG I/O board is equipped with 16 optically isolated binary inputs, eight optically isolated binary outputs, four 12-bit analog inputs, and two 12-bit analog outputs. There are several different Allen-Bradley 1771 series I/O modules. Allen-Bradley binary inputs can be DC voltage inputs, and TTL inputs. Binary outputs can be DC voltage outputs, TTL outputs, and contact closures. There is also the Allen-Bradley Redi-Panel which provides 16 binary input buttons, and 16 binary output lighted indicators for user configuration. The Allen-Bradley analog input and output modules may be either differential or single-ended in nature, and accurate to 12 bits.

\section{STANDARD TEMPERATURE, PRESSURE AND FLOW MONITORING}

Temperature, pressure and flow monitoring are usually done by display and signal conditioning units in close proximity to their relative sensors. These units supply a local display, and often a set of relay contact closures for triggering interlock situations. These units are procured from many different vendors, Omega, Love Controls, and Newport Electronics to name a few.
These units most often communicate to the control system by the output of a voltage or current level scaled to the temperature, pressure, or flow rate indicated on the display. These in turn can be read in as simple analog inputs, by the means discussed in the previous section. Other methods of communication include RS232, and RS485 interfaces. The RS232 units generally interface to the control system via BUGs. The RS485 units are linked together via the ir own multi-drop network. Although the current interface to these units is via BUGs, an interface directly to the $1 O C$ is in the planning stages.

\section{SUMMARY}

One of the Major features of EPICS is that it can be readily expanded to accommodate new devices. With the increase in number and sophistication of laboratory instruments, and the growing number of facilities using EPICS, the variety of devices supported under EPICS is currently increasing. The systems described in this paper in no way include all hardware supported under EPICS. Additional examples of device support under development include VXI devices and specific instrumentation for running synchrotron light source beam-line experiments. As more instruments are supported, one challenge to be faced will be to maintain a repository for making device support modules available to all EPICS users.

\section{REFERENCES}

[1] N.D. Arnold, G.J. Nawrocki, K.T. Daly, M.R. Kraimer, W.P. McDowell, "I/O Subnets for the APS Control System," Proceedings of the 1991 IEEE Particle Accelerator Conference, pp. 1496-1498, 1991.

[2] G.J. Nawrocki, N.D. Arnold, M.G. Hoffberg, J.R. Winans, S.J. Benes, "An I/O Subnet for the APS Control System The BITBUS Universal Gateway," Proceedings of the 1993 IEEE Particle Accelerator Conference, 1993.

[3] K.J. Coulter, R.A. Popper, D.D. Reid, S.J. Stein, L.R. Dalesio, C. Fite, M. Stettler, D. Warren, "Motor Control in the Experimental Physics and Industrial Control System," Submitted to the International Conference on Accelerator and Large Experimental Physics Control Systems, 1993.

$\uparrow$ BITBUS and iSBX are registered trademarks of the Intel Corporation and its affiliates.

\$ IndustryPack is a registered trademark of Green Spring Computers, Inc.

\section{DISCLAIMER}

\begin{abstract}
This report was prepared as an account of work sponsored by an agency of the United States Government. Neither the United States Government nor any agency thereof, nor any of their employees, makes any warranty, express or implied, or assumes any legal liability or responsibility for the accuracy, completeness, or usefulness of any information, apparatus, product, or process disclosed, or represents that its use would not infringe privately owned rights. Reference herein to any specific commercial product, process, or service by trade narıe, trademark, manufacturer, or otherwise does not necessarily constitute or imply its endorsement, recommendation, or favoring by the United States Government or any agency thereof. The views and opinions of authors expressed herein do not necessarily state or reflect those of the United States Government or any agency thereof.
\end{abstract}




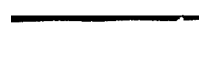

$$
\longrightarrow
$$

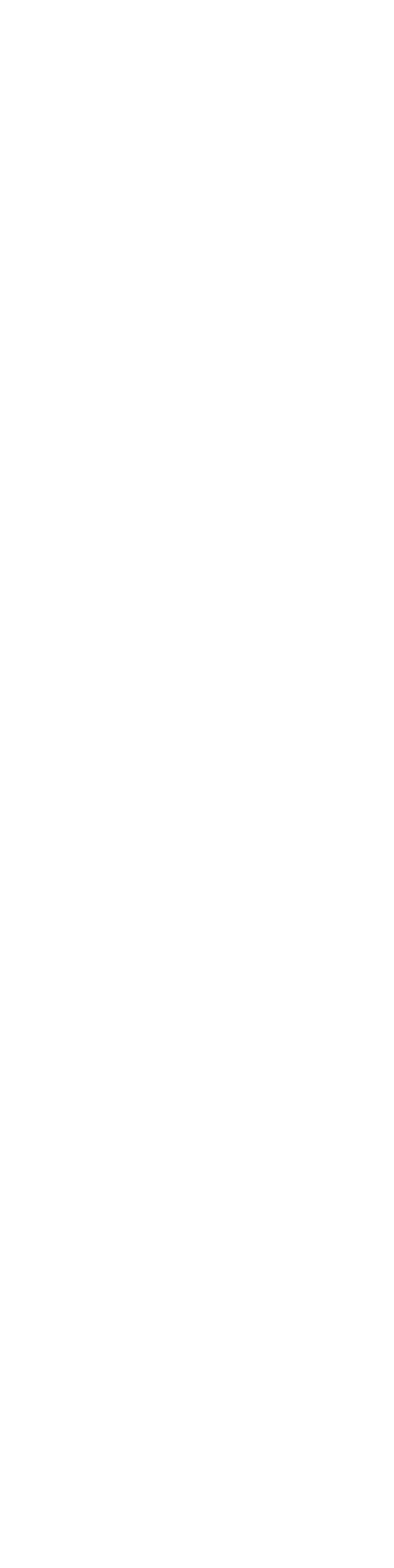

. 